\title{
Stimulated optomechanical excitation of surface acoustic waves in a microdevice
}

\author{
Gaurav Bahl', John Zehnpfennig',2, Matthew Tomes' \& Tal Carmon'
}

Stimulated Brillouin interaction between sound and light, known to be the strongest optical nonlinearity common to all amorphous and crystalline dielectrics, has been widely studied in fibres and bulk materials but rarely in optical microresonators. The possibility of experimentally extending this principle to excite mechanical resonances in photonic microsystems, for sensing and frequency reference applications, has remained largely unexplored. The challenge lies in the fact that microresonators inherently have large free spectral range, whereas the phase-matching considerations for the Brillouin process require optical modes of nearby frequencies but with different wave vectors. Here we rely on high-order transverse optical modes to relax this limitation and report the experimental excitation of mechanical resonances ranging from 49 to $1,400 \mathrm{MHz}$ by using forward Brillouin scattering. These natural mechanical resonances are excited in $100 \mu \mathrm{m}$ silica microspheres, and are of a surface-acoustic whispering-gallery type.

\footnotetext{
${ }^{1}$ Electrical Engineering and Computer Science, University of Michigan, Ann Arbor, Michigan 48109, USA. ${ }^{2}$ Electrical Engineering and Computer Science, United States Military Academy, West Point, New York 10996, USA. Correspondence and requests for materials should be addressed to G.B. (email: bahlg@umich.edu).
} 
$\mathrm{n}$ the past, interaction of optical and mechanical resonances in cavities arising from modulation of the optical round-trip length has allowed excitation of emission lines that were similar to those observed in molecular studies, though only originating from device vibrations ${ }^{1-4}$. Extensive research has since been performed towards extending other atomic-level effects, such as cooling ${ }^{5-7}$ and quantum effects ${ }^{8-12}$, to these optomechanical cavities. The ponderomotive forces used in these experiments $s^{1-3,5-7}$ have included centrifugal radiation pressure ${ }^{1}$, scattering force $\mathrm{c}^{5-7}$, and gradient force ${ }^{13}$. All of these experiments ${ }^{1-3,5-7,13}$ relied on the parametric optomechanical excitation of vibration (Fig. 1a). However, there is also a different path for cavity optomechanics, of exciting vibration through a stimulated process (Fig. 1b), associated with a quantum event involving the emission of a phonon into the mechanical resonance, which we will use here. Previously, the exploitation of stimulated Brillouin backscattering ${ }^{14,15}$ for stimulated photon-phonon emission has been deterred by either zero optical finesse in nanospheres ${ }^{16}$, or by negligible mechanical finesse in microspheres ${ }^{17}$. Fortunately, momentum conservation suggests ${ }^{18}$ that reversal of the scattering direction from backward ${ }^{14}$ to forward ${ }^{18,19}$, allows the generation of low-frequency phonons that suffer much less material dissipation ${ }^{20}$, resulting in high finesse acoustic modes. In the experiments that we report here, the stimulated process that excites the observed mechanical resonances is based on forward Brillouin scattering ${ }^{18,19}$ of photons from acoustic phonons.

Let us consider an optically stimulated travelling acoustic resonance (frequency $\Omega_{\mathrm{a}}$ ) on a microsphere device with an integer number of acoustical wavelengths $\left(M_{\mathrm{a}}\right)$ around the circumference (Fig. 1d, right). We further consider that this resonance travels uni-directionally along the equator at the speed of sound (Fig. 1b, left). The process responsible for the generation of this resonance is as follows-the travelling mechanical mode photoelastically writes an optical grating that is capable of scattering pump light. As the mechanical mode recedes at the speed of sound, it Doppler red-shifts the scattered light by the correct frequency to enter the Stokes optical mode $\left(\omega_{\mathrm{S}}=\omega_{\mathrm{p}}-\Omega_{a}\right)$. Simultaneously, the remaining pump-mode light along with the Doppler shifted Stokes-mode light can electrostrictively excite vibration at the beat frequency of the two optical modes. If this optically induced stress also travels at the speed of sound, the process described by photoelastic scattering and electrostriction becomes self sustaining. This intuitive phase-matching condition can be derived analytically by solving the coupled wave equations for mechanical and optical waves to reveal a synchronous solution ${ }^{20}$. The solution reveals that the propagation constant of the optical pump $M_{\mathrm{p}}$ (related to azimuthal $e^{i\left(M_{p} \cdot \phi-\omega_{p} \cdot t\right)}$ propagation) must equal the sum of the propagation constants of the resulting Stokes and acoustical modes $\left(M_{p}=M_{S}+M_{a}\right)$. This wave vector relation is associated with momentum conservation, as one pump photon is converted into a photon and a phonon, whose combined momentum must equal to that of the pump photon. This conservation consideration also suggests that low-rate vibrations that are forbidden in backward scattering ${ }^{15,17}$ processes (Fig. 1c, left) are allowable if forward scattering is utilized instead (Fig. 1c, right). Further, it is known that the material dissipation limit for phonons scales inversely with the square of frequency ${ }^{20}$. This suggests that scaling mechanical frequency from $11,000 \mathrm{MHz}$ (typical of backward scattering in silica) to $50 \mathrm{MHz}$ (this work) can potentially provide a 50,000-fold reduction in dissipation, transforming the stimulated acoustical wave ${ }^{14}$ into a high finesse mechanical resonance. Phase matching is challenging for forward scattering due to the requirement of having two optical modes with nearby frequencies but with propagation constants differing by $M_{\mathrm{a}}$. A degree of freedom that can help in achieving close frequencies for the $M_{\mathrm{p}}, M_{\mathrm{S}}$ modes is to change the transverse order of the mode till the optical frequencies are properly separated, as described in refs 18,21 .

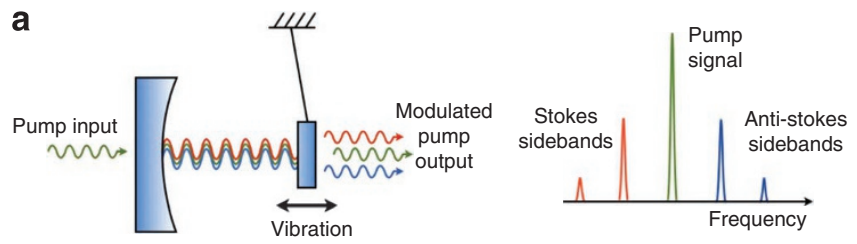

b

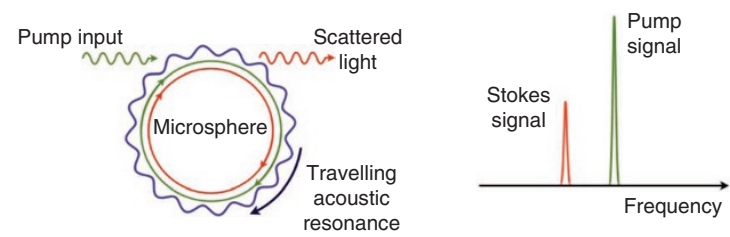

C

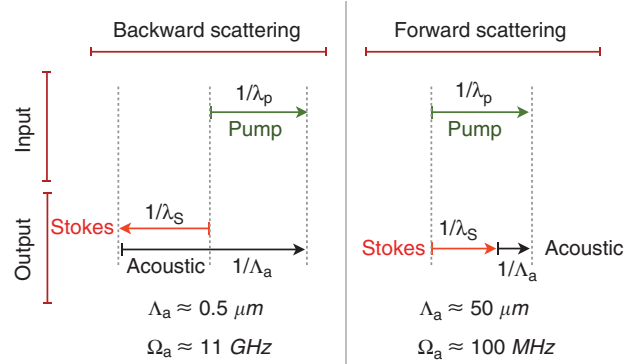

d

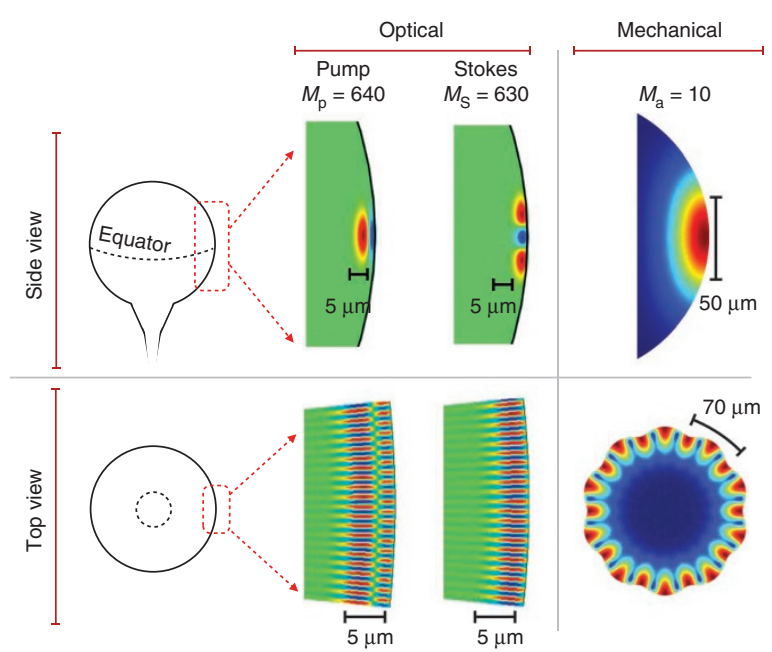

Figure 1 | Illustration for distinguishing stimulated cavity-optomechanics from parametric cavity-optomechanics. (a) In parametric excitation when one feeds the cavity with the laser right at the optical resonance, the pump is modulated due to a shifting optical resonance, resulting in two optical side bands accompanied by higher harmonics. If the laser is detuned, one can obtain asymmetric spectra to allow net energy transfer from light to the mechanical mode or vice versa. (b) In stimulated optomechanics (this work), the pump is scattered to only one Stokes sideband since the acoustic wave acts as a grating continuously moving away from the pump. (c) Wave vector conservation in forward Brillouin scattering leads to a much lower acoustic frequency regime when compared with backward Brillouin scattering. (d) Numerically solved optical and mechanical modes for phase-matched stimulated optomechanical interaction on a microsphere resonator, where colours represent electric field of the optical mode and the deformation of the mechanical mode. The mechanical mode (bottom-right) is a Rayleigh-type surface acoustic wave with experimentally observed frequency $57.8 \mathrm{MHz}$.

Here we experimentally demonstrate the excitation of acoustical modes of a silica microsphere by stimulated forward Brillouin scattering. The actuation mechanism is experimentally verified 
a
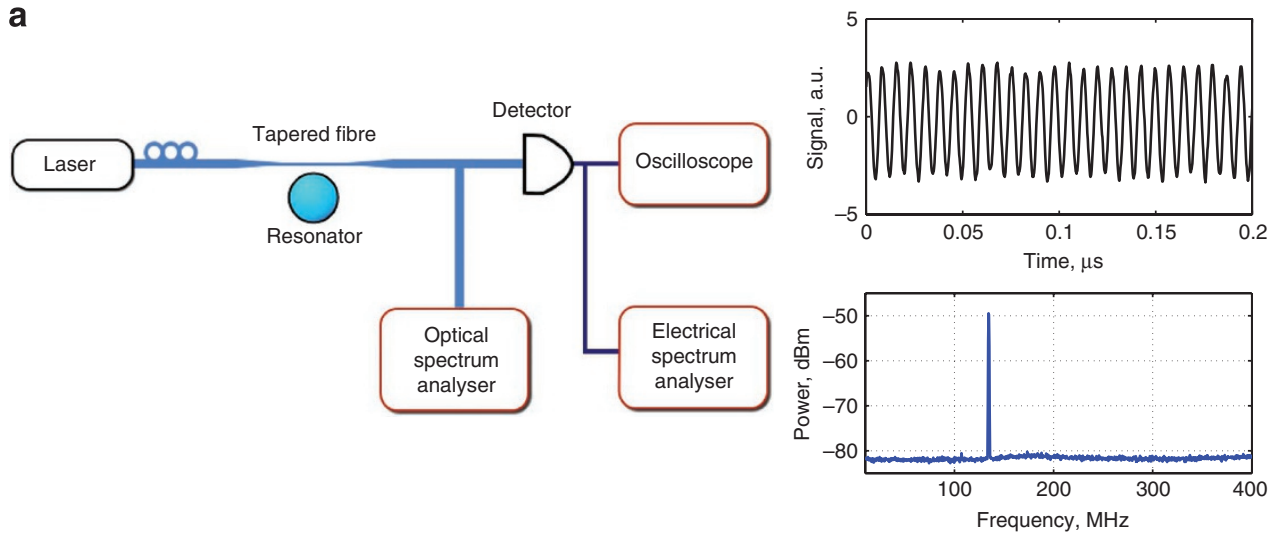

b

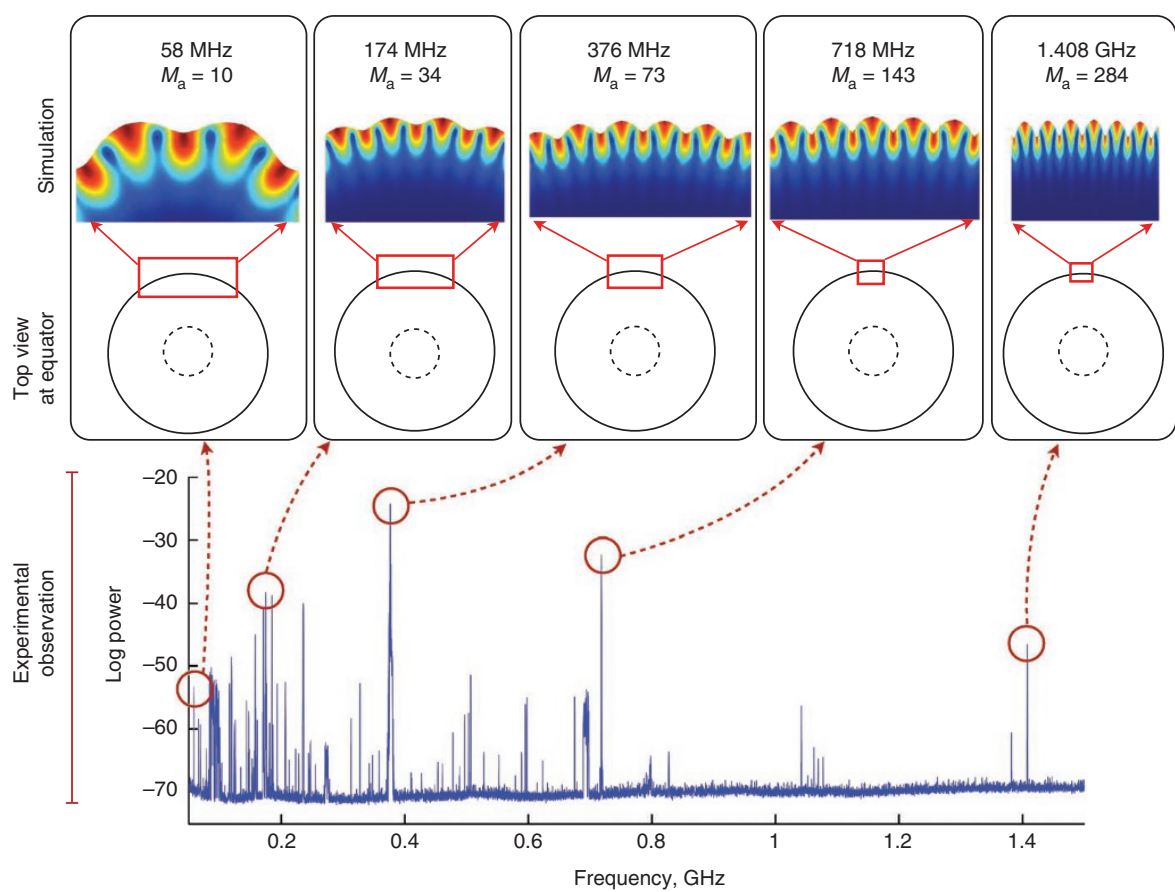

Figure 2 | Experimental setup and experimental modal spectroscopy. (a) The pump laser is coupled to the optical microsphere resonator through a tapered fibre. Forward-scattered optical signals and the pump transmission are subject to both optical and electrical spectrum analysis. The experimental data insets show an example $134 \mathrm{MHz}$ mechanical mode observed in electrical domain with an unchanging pump laser. (b) Many mechanical modes are observed when the pump laser is scanned slowly from 1,520-1,570 nm. We depict the best-match Rayleigh surface modes for several of the measured frequencies. Colours represent the deformation of the mechanical mode.

through observation of the optical signatures of the process. We measure acoustical frequencies ranging from $49 \mathrm{MHz}$ to $1,400 \mathrm{MHz}$. We expect our technique for exciting surface modes to be relevant for applications in surface-wave sensors and in oscillators.

\section{Results}

Modelling of optical and mechanical modes. We calculate a resonant trio (Fig. 1d) consisting of two optical modes and one mechanical mode, where the optical modes are calculated as shown in ref. 22 and the mechanical modes using FEM (see Methods). First, we set momentum to be conserved for the forward scattering process by choosing modes with azimuthal integer number of wavelengths along the circumference that obey $M_{\mathrm{p}}=M_{\mathrm{S}}+M_{\mathrm{a}}$ (for example, $640=$ $630+10)$. We then vary the transverse order of the optical modes until $\omega_{\mathrm{p}}=\omega_{\mathrm{S}}+\Omega_{\mathrm{a}}$ is met so that energy is conserved as well ${ }^{18,21}$. The mechanical mode that we calculate to fit here is a Rayleigh-type ${ }^{23}$ surface-acoustic-wave ${ }^{24}$ as indicated by the fact that particles move elliptically in the equatorial plane and by the fact that upon increasing
$M_{a}$ its speed asymptotically converges to the speed of a Rayleigh surface-acoustic-wave on planar substrates as given by the analytical solution $^{23}$. An acoustic frequency corresponding to the calculation of Figure $1 \mathrm{~d}$ will be experimentally measured in Figure $2 \mathrm{~b}$.

Measuring stimulated mechanical vibration. Mechanical vibration is stimulated by pumping the appropriate optical resonance of a microsphere using a tunable pump laser (see Methods, and Fig. 2a). The vibration is measured via the beating of the pump and Stokes modes on a photo detector. Temporal sinusoidal dynamics are observed (Fig. 2a, top right) together with the corresponding spectral line (Fig. 2a, bottom right) that we measure with an electrical spectrum analyser. We then use an optical spectrum analyser to verify that this beat indeed originates from a Stokes line that is separated from the pump by the acoustical frequency. Such correlation is shown in Figure 3. Additionally, a broadband spectrum analyser is used to verify that four-wave mixing ${ }^{25}$ or Raman lasing ${ }^{26}$ do not interfere with our measurements (see Methods). 

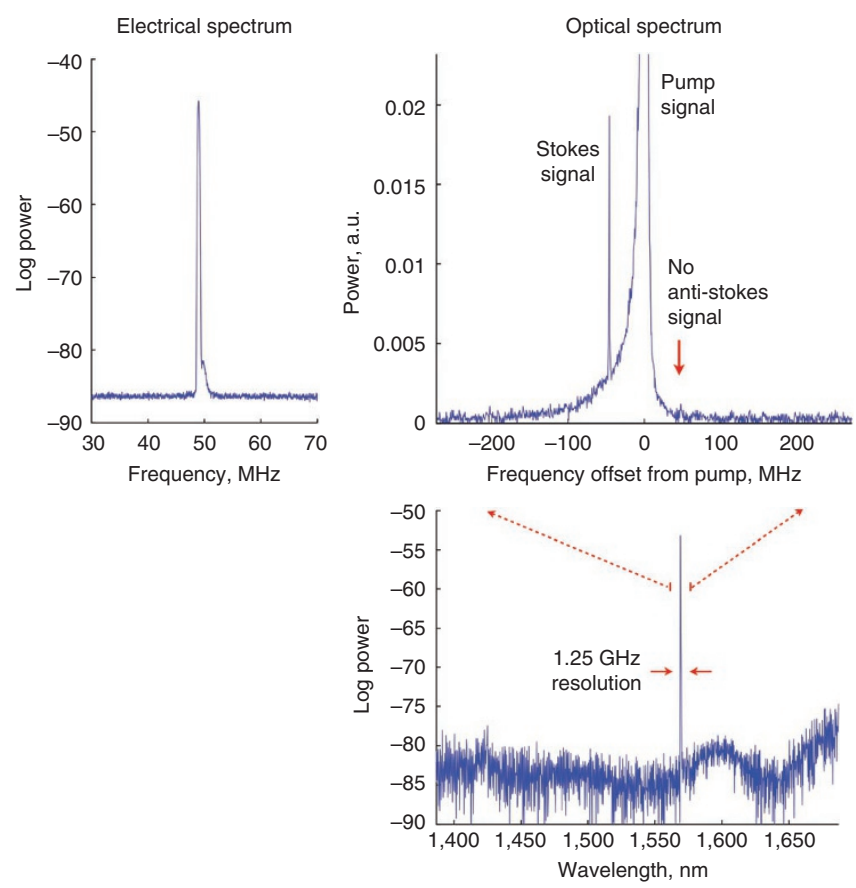

Figure 3 | Electrical and optical spectra for a $\mathbf{4 8 . 9} \mathbf{~ M H z ~ v i b r a t i o n . ~ S p e c t r a ~}$ were obtained in a sphere of radius $162 \mu \mathrm{m}$ with pump wavelength $1,567 \mathrm{~nm}$. The electrical spectrum represents the beat note between the two optical signals. The absence of the anti-Stokes sideband in the optical spectrum as well as the absence of higher harmonics clearly indicates that this is not a mechanical breathe mode. The wide optical spectrum (bottom) confirms that our observation is not disturbed by any other optical lines such as those arising from four-wave mixing or Raman scattering.

Modal spectroscopy measurement is performed by slowly tuning the laser through optical resonances while recording the vibration frequencies excited through resonant trios similar to what was calculated in Figure 1d, but having different modal orders. In the example shown in Figure 2b, we observed mechanical modes ranging from $58 \mathrm{MHz}-1.4 \mathrm{GHz}$ in a single sphere with the laser being tuned from $1,520-1,570 \mathrm{~nm}$. We depict several of these experimentally observed frequencies and provide their closest mechanical Rayleigh modes.

The vibration threshold is measured to be $22.5 \mu \mathrm{W}$ (Fig. $4 \mathrm{a}$ ) for a $320 \mu \mathrm{m}$ diameter sphere with quality factor $\sim 400$ million, pumped at $1.5 \mu \mathrm{m}$ wavelength, mechanically resonating at $175.36 \mathrm{MHz}$. As with other stimulated processes including Raman ${ }^{26}$ and Erbium ${ }^{27}$ microlasers, threshold power depends on the coupling distance between taper and resonator as measured in Figure $4 \mathrm{~b}$ and fitted to what is predicted by coupled-mode-theory analysis ${ }^{28}$ (see Methods). Qualitatively and to a certain limit, this threshold lowering behaviour is due to under-coupling reducing Stokes transmission out of the cavity and leaving more of it inside the resonator to provide electrostrictive forces that excite vibration.

Measuring the fine structure of the beat-note spectrum reveals a narrow and strong Lorentzian $(\sim 580 \mathrm{~Hz}$, Fig. 5a) superimposed over a weak and wide Lorentzian $(\sim 1 \mathrm{MHz}$, Fig. $5 \mathrm{~b}$ inset). The taller Lorentzian originates from the width of the vibration gain spectrum that is expected to be $\sim 2 \mathrm{kHz}$ wide ${ }^{20}$. In resonators such $2 \mathrm{kHz}$ width is expected to narrow ${ }^{18}$ while being limited by the Schawlow Townes relation ${ }^{29}$. The broad Lorentzian originates from the optical resonance width. It is worth mentioning that the stimulated process gain here is $\sim 1,000 \times$ narrower than the optical resonance ${ }^{20}$, in contrast with other processes ${ }^{17,26,27,30}$ where the relation is opposite. Interestingly, linewidths like in Figure 5a
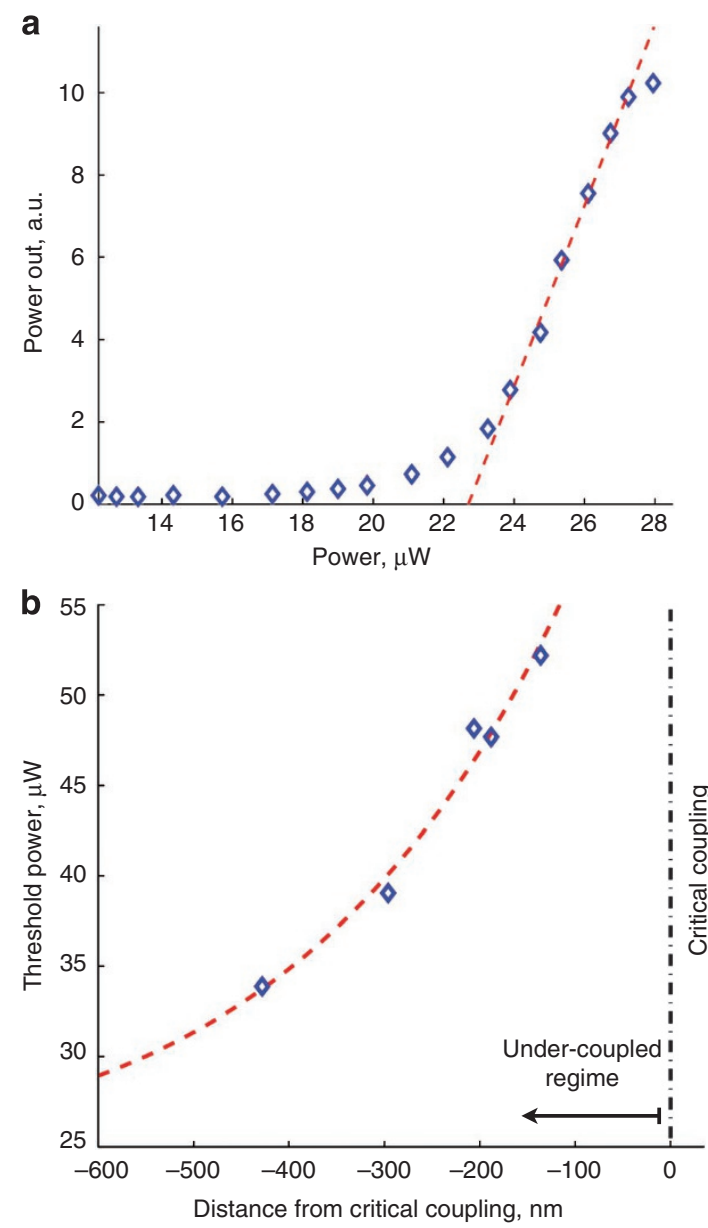

Figure 4 | Experimental observation of threshold power required for vibration. (a) Power in versus power out curve obtained for a $175.36 \mathrm{MHz}$ mechanical vibration. The linear fit line indicates $P_{\text {threshold }} \approx 22.5 \mu \mathrm{W}$. (b) Under-coupling reduces the threshold power. This data is obtained for a $134 \mathrm{MHz}$ mechanical mode. Dashed line is a fit to the theory presented in Methods.

are related to both the mechanical quality factor and the optical quality factor.

The mechanical finesse is estimated by measuring the vibration decay time. Although many additional factors interfere with this measurement, we attempted to perform a ring-down measurement by executing a fast scan of the pump wavelength. Based on several such scans we estimate a phonon lifetime $\tau \approx 40 \mu \mathrm{s}$, implying a mechanical quality factor of approximately $Q \approx 44,000$ (finesse 900) for the $175 \mathrm{MHz}$ mode shown in Figure $5 \mathrm{c}$. This is in comparison with material limited mechanical $Q \approx 48,000$ (ref. 20 ). In our experiments, the Stokes mode is produced at efficiencies of up to $50 \%$, with input power in the $\mathrm{mW}$ range.

\section{Discussion}

In this work, we report, for the first time, on the experimental stimulated optical excitation of high finesse mechanical resonances in silica microspheres via forward Brillouin scattering, indicating a new mechanism for studies in cavity optomechanics. The stimulated mechanical whispering gallery modes that are generated here are surface acoustic waves of the form typically excited using metal electrodes on piezoelectric substrates ${ }^{24}$. In our case, however, the electrodes are virtual, and are written by light travelling with the mechanical mode. 


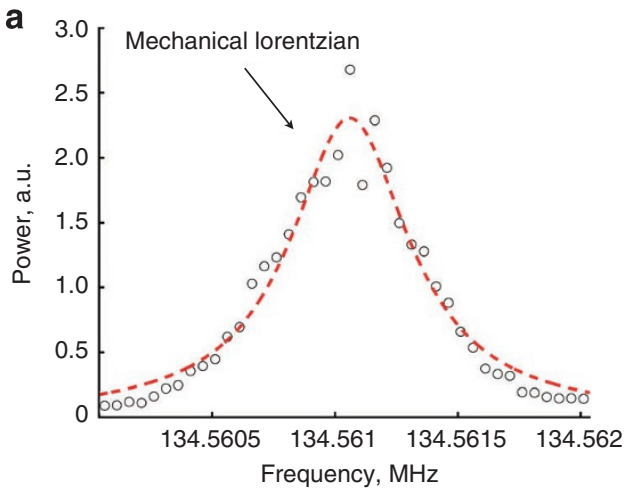

b
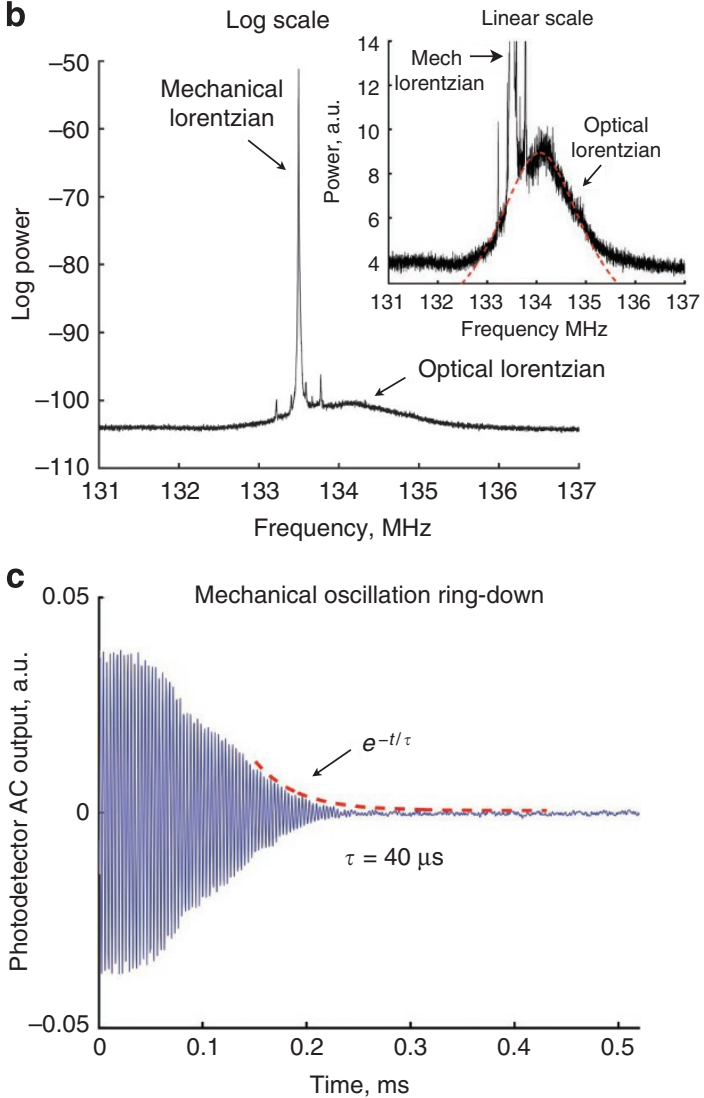

Figure 5 | Linewidth and mechanical mode characterization. (a) Sample beat linewidth captured for a $134 \mathrm{MHz}$ mode. The plotted Lorentzian guide indicates a FWHM linewidth of $580 \mathrm{~Hz}$. (b) The mechanical signal is superimposed on a low-power $\mathrm{MHz}$-width Lorentzian that originates from the optical resonance. (c) Vibration ring-down experiment for determining the mechanical quality factor of a $175 \mathrm{MHz}$ mechanical mode on a sphere of radius $R=162 \mu \mathrm{m}$.

The dissipation suffered by phonons at these mechanical resonances is near the material limit, since they are whisperinggallery modes that propagate azimuthally just like the optical whispering gallery modes that excite them. Such concentration of vibration at the sphere surface removes the necessity for notch supports and mechanical quarter-wavelength leakage isolators for the resonator, simplifying the mechanical design of the system. This is unlike the case of breathing modes ${ }^{4}$ that have propagation components in the direction of the mechanical supporting structures. Additionally, the mechanism of electrostriction that supports the stimulated Brillouin process, is common to all dielectrics inclusive of every amorphous or crystalline optical material. Such Brillouin amplification benefits from having the highest gain of all the optical nonlinearities ${ }^{20}$. In silica, for instance, Brillouin gain is more than two orders of magnitude higher than Raman gain ${ }^{20}$.

Microresonators that are presently at the core of many experiments in optics for enhancing electromagnetic fields ${ }^{31,32}$ are shown here to allow stimulated optical excitation of high finesse whispering gallery mechanical resonances. The narrow oscillation linewidth, and the fact that the mechanical mode is concentrated to within one acoustical wavelength from the interface with the environment make this device attractive for sensing changes in the environment ${ }^{33}$. Further, these mechanical resonances see dissipation near the material-property limit, and are consequently very useful in oscillator applications as well.

\section{Methods}

Numerical modelling of optical and mechanical modes. The mechanical modes that we experimentally observed were identified using three-dimensional finite element modelling in COMSOL Multiphysics ${ }^{34}$. The existence of pairs of optical modes that satisfy phase match to excite these mechanical modes was confirmed using the numerical method described in ref. 22. Gross tuning of phase match was achieved by discretely changing the transverse order of the optical modes. Fine tuning was then performed by modifying the ellipticity of the sphere till exact phase match was reached. Shape and size of the calculated device were kept within the measurement error using our microscope.

Experimental setup. Our experimental setup (Fig. 2a) consists of a silica microsphere resonator ${ }^{35}$ (typical optical $Q>10^{8}$ ) evanescently coupled to a telecom-wavelength laser $(\sim 1,550 \mathrm{~nm})$ through a tapered optical fibre ${ }^{36-38}$. We tune the pump laser to one of the optical resonances, and via the other end of the taper, measure the output of the partially transmitted pump along with the Stokes mode that vibration scatters. Optical spectrum analysers are used to verify optical signals, whereas photodetectors provide the electrical beat signal. We emphasize that vibration is stimulated with a continuous in-time single frequency laser, and that no external oscillator, controller, or feedback is used.

Verification of optical signals. The presence of a single Stokes side-band to the optical pump is confirmed by optical spectrum analysis through a Thorlabs SA200-14A tunable Fabry-Perot spectrum analyser (Fig. 3, top). We confirm that no optical lines other than the stimulated Stokes line exist in our system by using a second OSA (Advantest Q8384 Optical Spectrum analyzer) that covers a broad spectral range (Fig. 3, bottom). Also, as expected, the broad span OSA cannot resolve the two optical signals due to their close spacing.

Derivation for coupling-dependent threshold. The relationship of coupling threshold to the coupling distance can be derived following the rate equations in ref. 39. Starting with the non-depleted pump approximation, a rate equation for the pump mode can be obtained

$$
\dot{A}_{\mathrm{p}}=i \cdot C(x) \cdot B_{\mathrm{in}}-\left(\delta_{\mathrm{c}}(x)+\delta_{\mathrm{o}}\right) \cdot A_{\mathrm{p}}
$$

where $A_{\mathrm{p}}$ is the field amplitude of the pump mode, $B_{\text {in }}$ is the pump field amplitude in the coupling waveguide, $x$ is taper-sphere distance, $C(x)$ is the taper-sphere distance-dependent coupling coefficient, $\delta_{o}$ is the intrinsic loss, and $\delta_{c}(x)$ is the coupling loss. From here, the steady state expression,

$$
A_{\mathrm{p}}=\frac{i \cdot C(x) \cdot B_{\text {in }}}{\delta_{\mathrm{c}}(x)+\delta_{\mathrm{o}}}
$$

can be obtained. The Stokes mode rate equation is derived in a manner similar to equation (1).

$$
\dot{A}_{\mathrm{s}}=-g \cdot\left|A_{\mathrm{p}}\right|^{2} \cdot A_{\mathrm{s}}-\left(\delta_{\mathrm{c}}(x)+\delta_{\mathrm{o}}\right) \cdot A_{\mathrm{s}}
$$

where $g$ is the process gain and $A_{\mathrm{s}}$ is the Stokes mode field amplitude. By balancing gain and loss terms in equation (3) and incorporating equation (2), the threshold power can be obtained.

$$
B_{\text {in }}^{2}=\frac{\left(\delta_{\mathrm{c}}(x)+\delta_{\mathrm{o}}\right)^{3}}{g \cdot C(x)^{2}}
$$

This is rewritten as

$$
P_{\text {threshold }} \propto \frac{\left(T(x)^{2} / 2 \tau_{0}+\delta_{0}\right)^{3}}{g \cdot T(x) / \tau_{0}}
$$

where $T(x)$ is the coefficient of transmittance exponentially related to $x$ the tapersphere distance, and $\tau_{0}$ is optical round-trip time. 


\section{References}

1. Carmon, T., Rokhsari, H., Yang, L., Kippenberg, T. \& Vahala, K. Temporal behavior of radiation-pressure-induced vibrations of an optical microcavity phonon mode. Phys. Rev. Lett. 94, 223902 (2005).

2. Rokhsari, H., Kippenberg, T., Carmon, T. \& Vahala, K. Radiation-pressuredriven micro-mechanical oscillator. Opt. Express 13, 5293-5301 (2005).

3. Kippenberg, T. J., Rokhsari, H., Carmon, T., Scherer, A. \& Vahala, K. J. Analysi of radiation-pressure induced mechanical oscillation of an optical microcavity. Phys. Rev. Lett. 95, 033901 (2005).

4. Carmon, T. \& Vahala, K. J. Modal spectroscopy of optoexcited vibrations of a micron-scale on-chip resonator at greater than $1 \mathrm{GHz}$ frequency. Phys. Rev. Lett. 98, 123901 (2007)

5. Arcizet, O., Cohadon, P.- F., Briant, T., Pinard, M. \& Heidmann, A. Radiationpressure cooling and optomechanical instability of a micromirror. Nature 444, 71-74 (2006).

6. Gigan, S. et al. Self-cooling of a micromirror by radiation pressure. Nature 444 67-70 (2006).

7. Kleckner, D. \& Bouwmeester, D. Sub-kelvin optical cooling of a micromechanical resonator. Nature 444, 75-78 (2006).

8. Schwab, K. C. \& Roukes, M. L. Putting mechanics into quantum mechanics. Phys. Today 58, 36-42 (2005).

9. Vitali, D. et al. Optomechanical entanglement between a movable mirror and a cavity field. Phys. Rev. Lett. 98, 030405 (2007).

10. Wilson-Rae, I., Nooshi, N., Zwerger, W. \& Kippenberg, T. J. Theory of ground state cooling of a mechanical oscillator using dynamical backaction. Phys. Rev. Lett. 99, 093901 (2007).

11. Marquardt, F., Chen, J. P., Clerk, A. A. \& Girvin, S. M. Quantum theory of cavity-assisted sideband cooling of mechanical motion. Phys. Rev. Lett. 99, 093902 (2007)

12. Thompson, J., Zwickl, B., Jayich, A., Marquardt, F., Girvin, S. \& Harris, J. Strong dispersive coupling of a high-finesse cavity to a micromechanical membrane. Nature 452, 72-75 (2008).

13. Povinelli, M., Johnson, S. \& Joannopoulos, J. Slow-light, band-edge waveguides for tunable time delays. Opt. Express 13, 7145-7159 (2005).

14. Chiao, R. Y., Townes, C. H. \& Stoicheff, B. P. Stimulated brillouin scattering and coherent generation of intense hypersonic waves. Phys. Rev. Lett. 12, 592-595 (1964).

15. Grudinin, I. S., Matsko, A. B. \& Maleki, L. Brillouin lasing with a $\mathrm{CaF}_{2}$ whispering gallery mode resonator. Phys. Rev. Lett. 102, 043902 (2009).

16. Kuok, M. H., Lim, H. S., Ng, S. C., Liu, N. N. \& Wang, Z. K. Brillouin study of the quantization of acoustic modes in nanospheres. Phys. Rev. Lett. 90, 255502 (2003).

17. Tomes, M. \& Carmon, T. Photonic micro-electromechanical systems vibrating at X-band (11-GHz) rates. Phys. Rev. Lett. 102, 113601 (2009).

18. Matsko, A. B., Savchenkov, A. A., Ilchenko, V. S., Seidel, D. \& Maleki, L. Optomechanics with surface-acoustic-wave whispering-gallery modes. Phys. Rev. Lett. 103, 257403 (2009).

19. Shelby, R., Levenson, M. \& Bayer, P. Resolved forward Brillouin scattering in optical fibers. Phys. Rev. Lett. 54, 939-942 (1985).

20. Boyd, R. W. Nonlinear Optics 3rd edn, Chapter 9. Elsevier, 2008.

21. Carmon, T. \& Vahala, K. Visible continuous emission from a silica microphotonic device by third-harmonic generation. Nat. Phys. 3, 430-435 (2007)

22. Oxborrow, M. Traceable 2-D finite-element simulation of the whisperinggallery modes of axisymmetric electromagnetic resonators. IEEE Trans. Microwave Theory Tech. 55, 1209-1218 (2007).
23. Rayleigh, L. On waves propagated along the plane surface of an elastic solid. Proc. R. Soc. Lond. A17, 4-11 (1885).

24. Campbell, C. Surface Acoustic Wave Devices for Mobile and Wireless Communications 1st edn,Academic Press, 1998.

25. Kippenberg, T. J., Spillane, S. M. \& Vahala, K. J. Kerr-nonlinearity optical parametric oscillation in an ultrahigh-q toroid microcavity. Phys. Rev. Lett. 93, 083904 (2004).

26. Spillane, S., Kippenberg, T. \& Vahala, K. Ultralow-threshold raman laser using a spherical dielectric microcavity. Nature 415, 621-623 (2002).

27. Min, B., Yang, L. \& Vahala, K. Controlled transition between parametric and Raman oscillations in ultrahigh-Q silica toroidal microcavities. Appl. Phys. Lett. 87, 181109 (2005).

28. Haus, H. A. \& Huang, W. Coupled-mode theory. Proc. IEEE 79, 1505-1518 (1991)

29. Schawlow, A. L. \& Townes, C. H. Infrared and optical masers. Phys. Rev. 112, 1940-1949 (1958).

30. Vahala, K. J. Back-action limit of linewidth in an optomechanical oscillator. Phys. Rev. A 78, 023832 (2008).

31. Vahala, K. J. Optical Microcavities, World Scientific Publishing, 2005

32. Chang, R. K. Optical Processes in Microcavities, World Scientific Publishing, 1996

33. Ballantine, D. et al. Acoustic Wave Sensors: Theory, Design, \& Physico-Chemical Applications (Applications of Modern Acoustics), Academic, 1996.

34. Comsol Group. COMSOL Multiphysics, COMSOL, Ab., (online) Available at: http://www.comsol.com/.

35. Gorodetsky, M. \& Ilchenko, V. High-Q optical whispering-gallery microresonators: precession approach for spherical mode analysis and emission patterns with prism couplers. Opt. Commun. 113, 133-143 (1994).

36. Cai, M. \& Vahala, K. Highly efficient hybrid fiber taper coupled microsphere laser. Opt. Lett. 26, 884-886 (2001).

37. Spillane, S., Kippenberg, T., Painter, O. \& Vahala, K. Ideality in a Fiber Taper-Coupled Microresonator System for Application to Cavity Quantum Electrodynamics. Phys. Rev. Lett. 91, 043902 (2003).

38. Knight, J. C., Cheung, G., Jacques, F. \& Birks, T. A. Phase-matched excitation of whispering-gallery-mode resonances by a fiber taper. Opt. Lett. 22, 1129-1131 (1997).

39. Gorodetsky, M. L. \& Ilchenko, V. S. Optical microsphere resonators: optimal coupling to high-Q whispering-gallery modes. J. Opt. Soc. Am. B 16, 147-154 (1999).

\section{Acknowledgements}

The authors acknowledge discussions with C.X. Deng, Y.-S. Hsiao, and A.B. Matsko. This work was supported by the DARPA ORCHID program through a grant from AFOSR.

\section{Author contributions}

All the authors jointly designed and conceived the experiments. G.B. and J.Z. built the setup, fabricated the devices, and carried out the experiments. M.T., G.B. and J.Z. performed the analytical and numerical calculations. T.C. supervised all aspects of this project.

\section{Additional information}

Competing financial interests: The authors declare no competing financial interests.

Reprints and permission information is available online at http://npg.nature.com/ reprintsandpermissions/

How to cite this article: Bahl, G. et al. Stimulated optomechanical excitation of surface acoustic waves in a microdevice. Nat. Commun. 2:403 doi: 10.1038/ncomms1412 (2011). 